\title{
Mesa redonda
}

\section{Los Centros de Atención Infantil Temprana (CAIT): una red de centros y profesionales}

\author{
A. Montes Lozano \\ Centro de Atención Infantil Temprana San Rafael, Granada. España.
}

\section{INTRODUCCIÓN}

Aproximadamente han transcurrido casi cuatro décadas desde que se crearan en nuestro país los primeros Centros de Atención Temprana, denominados por la nomenclatura de la época centros de estimulación precoz. Con la necesidad de crear un modelo de funcionamiento homogéneo se constituyó el Grupo de Atención Temprana (GAT) que elaboró el Libro Blanco de la Atención Temprana ${ }^{1}$ (2000) cuyo documento destaca la importancia de la interdisciplinariedad, la globalidad, la coordinación y la calidad, convirtiéndose en un referente para todos los que trabajamos en este ámbito.

Durante estos años se ha producido una relativa consolidación de estos servicios, a pesar de haber sufrido una constante evolución a lo largo de su trayectoria histórica. Algunos de estos cambios han sido motivados por la propia evolución del concepto de discapacidad (íntimamente ligado a los Servicios de Atención Temprana) que ha ido desde una perspectiva centrada en el niño hacia una perspectiva centrada en el ambiente. Los problemas del desarrollo pasan de ser considerados un rasgo absoluto del individuo, a ser el resultado de la interacción entre la persona con discapacidad y su entorno. Por ello, hemos pasado de un modelo de intervención centrado en el niño a un modelo que tiene en cuenta al niño, a su familia y a los distintos contextos en los que tiene lugar el desarrollo.

\section{DEFINICIÓN DE CENTROS DE ATENCIÓN INFANTIL TEMPRANA}

Los Centros de Desarrollo Infantil y Atención Temprana (CDIAT), en Andalucía denominados Centros de Atención Infantil Temprana (CAIT), son los servicios autónomos donde se proporciona atención ambulatoria individualizada y que tienen por objetivo ofrecer un conjunto de intervenciones dirigidas a la población infantil (de cero a seis años), a su familia y a su entorno, con la finalidad de dar respuesta lo antes posible a las necesidades que presentan niños con alteraciones en el desarrollo o que tienen riesgo de padecerlas.

Estas intervenciones deben contemplar la globalidad del niño, y han de ser planificadas por un equipo de profesionales de orientación interdisciplinar o transdisciplinar. Este último concepto hace referencia a los equipos donde sus componentes adquieren conocimientos de otras disciplinas relacionadasy las incorporan a su práctica. Normalmentem los CAIT suelen estar formados por profesionales de distintas disciplinas: psicólogos, logopedas, fisioterapeutas, maestros, trabajadores sociales, terapeutas ocupacionales, etc. 
El principal objetivo de un CAIT es poner en marcha los recursos necesarios para que esta población reciba, siguiendo un modelo que considere los aspectos biopsicosociales, todo aquello que desde la vertiente preventiva y terapéutica pueda potenciar su capacidad de desarrollo y de bienestar, facilitando de esta manera su integración en el medio familiar, escolar y social, así como su autonomía personal. En resumen, la actividad del Equipo de Atención Temprana se enmarca sobre todo en el límite de entrada de la Prevención Terciaria.

En Andalucía existen dos tipos de CAIT.

- CAIT generalistas: atienden a niños/as menores de seis años que presenten un trastorno del desarrollo o que tengan riesgo de padecerlo.

- CAIT específicos: en ellos solo se atiende a niños/as que sufran una patología concreta (por ejemplo, trastornos del espectro autista, discapacidad auditiva, discapacidad visual, etc.). Poseen mayor grado de especialización y suelen estar ubicados en asociaciones.

\section{PROCESO ASISTENCIAL EN UN CENTRO DE ATENCIÓN INFANTIL TEMPRANA}

Los Centros de Atención Temprana en nuestro país dependen de distintas Consejerías (Salud, Educación o Bienestar Social) en las diferentes comunidades autónomas. Se hace, por lo tanto, complicado dar un modelo de CAIT, ya que estos obedecen a los decretos y acuerdos que existen en cada comunidad.

Para facilitar un mejor conocimiento de la actividad que se realiza en un CAIT, a continuación se describen las distintas fases del proceso asistencial que se suele seguir.

Dado que los requisitos y mecanismos de acceso a los Centros de Atención Temprana en nuestro país son diversos, me referiré en los puntos donde exista esa variabilidad a la realidad de Andalucía.

\section{Entrada al proceso}

Se produce siempre y cuando el pediatra de Atención Primaria realiza una derivación al CAIT de referencia, debido bien a la existencia de un trastorno del desarrollo, bien a la sospecha o riesgo de padecerlo. La detección de un problema en el curso del desarrollo puede hacerla el mismo pediatra, la familia, la escuela infantil o cualquier entorno.

\section{Acogida}

La recepción de ambos padres, a ser posible, y del menor se hace en el centro. En ella se suministra información a la familia sobre en qué consiste el CAIT, cuál es el sentido y la filosofía de la atención temprana y qué recursos existen a su disposición. A su vez, se firma un consentimiento informado que hace de carta de derechos y deberes.

\section{Valoración}

La entrevista inicial y la valoración del menor propiamente dicha son realizadas por el equipo de valoración. Es frecuente hacerlas el mismo día de la acogida. Tienen como misión la recogida de información, y la evaluación y valoración de las problemáticas y necesidades planteadas, tanto del niño como de la familia.

En la entrevista semiestructurada inicial se suele recoger una anamnesis personal y familiar del caso y en ella se incluyen el motivo de consulta, los datos personales, los antecedentes familiares, los datos pre-, peri- y posnatales, el desarrollo psicoevolutivo del menor, los datos sobre la escolarización, la información sobre el grado de autonomía en las actividades básicas de la vida diaria, los estilos de crianza, etc. Asimismo, se recaba información sobre otros recursos sanitarios y/o educativos que pueda estar recibiendo el niño. Para valorar a la familia también podemos servirnos de herramientas como el Cuestionario PSI (Parenting Stress Index) ${ }^{2}$.

Posteriormente, se evalúa al niño para obtener un perfil de capacidades que nos permita detectar los puntos débiles y fuertes sobre los cuales asentar la intervención, y a su vez establecer una primera 
aproximación diagnóstica que es codificada sobre la base de la Organización Diagnóstica para la Atención Temprana (ODAT). Para dicha evaluación, se suelen emplear test estandarizados y la observación en tareas semiestructuradas. En estas edades la evaluación debe ser muy flexible, pues tenemos que garantizar la colaboración del menor y muchos de los test estandarizados poseen una rigidez en su administración que es menos aconsejable. Las pruebas estandarizadas se dividen en:

- Escalas de desarrollo. Entre las más empleadas están la Escala de desarrollo Psicomotor BrunetLezine ${ }^{4}$ Revisada, el Inventario de desarrollo Battelle o la Guía Portage.

- Test de aptitudes generales. Los más utilizados son el Escala de inteligencia de Wechsler para preescolar y primaria WPPSI-IIII, la Escala de aptitudes y psicomotricidad de McCarthy ${ }^{6}$, etc.

- Test específicos. Test de vocabulario en imágenes Peabody, Test de desarrollo de la percepción visual Frostig ${ }^{7}$, etc.

Frecuentemente, complementamos la información recogida a los padres y la obtenida en la evaluación con cuestionarios o preguntas administrados a profesores o a otras figuras de referencia en la vida del niño que puedan aclarar aún más sobre su desarrollo.

Una vez se establece una hipótesis diagnóstica y se tiene un perfil de desarrollo del menor, se elabora un plan de actuación donde se recoge el tipo de intervención que va a recibir y el número de sesiones semanales, que puede oscilar entre uno y tres. Toda esta información se recoge en un informe escrito y es devuelta a la familia adaptándola a la gravedad del diagnóstico, lo novedoso que sea para ellos y la capacidad para comprenderlo y asumirlo, realizando en los casos necesarios una contención emocional que pretende sostener y aliviar psicológicamente a la familia del impacto emocional producido por el descubrimiento de un hijo con discapacidad. De hecho, uno de los principales objetivos en estos momentos será conseguir que los padres acepten la realidad de su hijo, que aprendan a amarlo por ser lo que es y no a pesar de serlo.
Consideramos que dicha aceptación es el punto de arranque que facilitará y potenciará todo el proceso posterior ${ }^{3}$. Por ello, debemos proporcionar a los padres las estrategias y recursos adecuados para lograr la confianza en sus propias posibilidades que, con frecuencia, se ven distorsionadas por el impacto del diagnóstico.

\section{Intervención terapéutica}

Consiste en la ejecución del plan de actuación. Implica la elaboración de un diseño de actuación específico con objetivos y pautas adaptadas a cada niño en cada una de las áreas del desarrollo evolutivo (cognición, comunicación y lenguaje, motricidad, conducta adaptativa y social, etc.). Estos programas se concretan en un documento que recoge una serie de objetivos a corto, medio y largo plazo con una metodología de intervención, y que suele ser explicado a las familias dotándolas de la información y formación necesarias para que puedan ser igualmente implementados por ellas en un entorno más ecológico. El programa, por lo tanto, debe incluir tanto objetivos y/o actividades de intervención estructurada en contextos terapéuticos, como objetivos y actividades pautadas para los entornos naturales.

Las sesiones de tratamiento en un CAIT suelen ser individuales, excepto en aquellos casos donde parte de la intervención sea potenciar aspectos sociales y de comunicación entre iguales. En esos casos se harán sesiones conjuntas con el consentimiento de los padres o tutores de los niños implicados. La duración de las sesiones oscila entre los 40 minutos y la hora de duración, dependiendo del CAIT. En este tiempo, el terapeuta trabaja con el niño y los padres, proporciona pautas y asesoramiento sobre el manejo de ciertas situaciones e informa de los progresos y de cambios que se vayan produciendo en el plan de actuación. Es imprescindible promover la participación activa de la familia en el proceso de desarrollo del niño y en la toma de decisiones que se vayan tomando.

De forma regular se evalúa la eficacia del tratamiento a través de la observación cualitativa y la 
administración de escalas de desarrollo. Los distintos tipos de tratamiento (por ejemplo, cognitivo, logopédico, fisioterapéutico, etc.) son llevados a cabo por diferentes perfiles profesionales procedentes de distintas disciplinas. Esto exige una adecuada coordinación entre los componentes del equipo. Por eso se llevan a cabo reuniones de equipo con una temporalización variable dependiendo del CAIT, pero que facilitan la coordinación y el avance hacia esa transdisciplinariedad antes mencionada.

Otra modalidad de intervención es el seguimiento. Los niños que pasan a esta modalidad acuden al CAIT de forma mensual, trimestral o semestral y son atendidos normalmente por el psicólogo que realizó la fase de valoración. A esta modalidad ingresan los niños por las siguientes razones:

- Debido a la levedad de los síntomas, puede asegurarse un óptimo desarrollo con solo proporcionar una serie de pautas a las familias.

- El niño no muestra aún necesidades de intervención, pero por los factores de riesgo que presenta se prevé que dichas necesidades aparezcan, por lo que se mantiene en seguimiento para detectarlas de forma precoz.

- El niño ha estado en la modalidad de tratamiento y se ha obtenido cierta normalización; sin embargo, es recomendable seguir pautando a la familia en algunos aspectos para evitar la aparición de nuevas dificultades.

Al finalizar el tratamiento, cuando se le va a dar el alta al niño, se procede generalmente a una última evaluación, que será reflejada en el informe de alta. Esta información es de gran utilidad para los profesionales que continuarán trabajando con el niño desde otros servicios.

Dentro de todo este proceso, se realizan otras actuaciones con el objetivo de atender integralmente las necesidades del niño y su familia. Estas otras actuaciones son las siguientes:

\section{Escuela de padres/madres}

Son actividades dirigidas a facilitar información y asesoramiento a las familias y se tratan los temas de mayor interés para ellos (modificación de conducta, estimulación del lenguaje en el hogar, control de esfínteres, seminarios sobre distintas patologías, etc.).

\section{Coordinación interinstitucional}

Reuniones con los distintos agentes que intervienen o tienen un papel importante en el desarrollo del menor (por ejemplo, maestros, profesionales del ámbito privado, neuropediatras, etc.). El objetivo de estas reuniones es la mayor coordinación posible en las intervenciones que se llevan a cabo, así como gestionar adecuadamente los recursos sanitarios, educativos y sociales que recibe cada niño. En este sentido, se hace imprescindible lograr una correcta continuidad de la atención que reciben nuestros niños en la próxima etapa que es el colegio, promoviendo un marco común y que las familias no vivan el alta en el CAIT como un punto y final, sino como un punto y seguido. Esto se ha facilitado en Andalucía mediante el sistema informático Alborada, que permite establecer esa relación entre distintas Consejerías.

\section{Formación externa}

Igualmente, los CAIT también participan en actividades de formación/orientación dirigidas a las familias y a los profesionales de los distintos sectores implicados, principalmente educativo y sanitario, con respecto a las necesidades y características del desarrollo infantil y los signos tempranos de los distintos trastornos del neurodesarrollo.

\section{Salida del proceso}

Los criterios de alta que generalmente se siguen en los CAIT son:

- Edad: el menor cumple seis años.

- Confirmación de un desarrollo adecuado: se produce una evolución satisfactoria hacia la normalización en el desarrollo.

- Cambio de equipo responsable, es decir, pasa a atender las necesidades del niño otro servicio o institución, como pueden ser los apoyos pres- 
tados desde el centro educativo con los maestros de Pedagogía Terapéutica o de Audición y Lenguaje.

- Alta voluntaria.

- Fallecimiento.

Para finalizar, y aunque somos conscientes de que la situación economica actual en ocasiones no favorece la calidad de nuestra practica profesional, ya que nos encontramos ratios desmesuradas, falta de recursos, etc, debemos comprometernos a ofrecer unas prácticas profesionales que, superando todo esto, garanticen la calidad de todas nuestras acciones. Se hace importante implantar una cultura de calidad en lugar de un simple cumplimiento de la normativa. Para asegurar una atención de calidad es imprescindible satisfacer las necesidades de formación de los profesionales que forman parte de los CAIT. Es cierto que cada centro tiene su idiosincrasia y un marco teórico que le influye, pero es necesario que nuestros profesionales estén bien formados y empleen enfoques y estrategias de intervención con una buena evidencia empírica. Para avanzar en los conceptos de eviden-

\section{BIBLIOGRAFÍA}

1. Federación Estatal de Asociaciones de Profesionales de Atención Temprana (GAT). Libro Blanco de la Atención Temprana (2000). Real Patronato sobre Discapacidad.

2. Abidin R. Parenting Stress Index (PSI), 3.ㄹ ed. Odessa Florida: Psychological Assesments Resources; 1995.

3. De Linares C. Ministerio de Trabajo y Asuntos Sociales. INSERSO. El papel de la familia en Atención Temprana. Minusval. 2003;67-70.

4. Brunet O, Lezine I. Escala de desarrollo psicomotor de la primera infancia Brunet-Lezine Revisada. PSYMTEC; 1997. cia científica en Atención Temprana, obligatoriamente tenemos que introducir la investigación dentro de las prácticas de nuestros equipos profesionales.

Otros indicadores de calidad tienen que ver con la gestión del centro, su accesibilidad, disponer de un equipo interdisciplinar bien coordinado, proporcionar la correcta información a las familias sobre el plan de actuación para facilitar así la toma de decisiones compartidas, etc.

Para favorecer e impulsar estos procesos de mejora continua, la Agencia de Calidad Sanitaria de Andalucía ${ }^{8}$ ha creado un documento donde se reflejan los estándares de calidad para los CAIT.

\section{CONFLICTO DE INTERESES}

El autor declara no presentar conflictos de intereses en relación con la preparación y publicación de este artículo.

\section{ABREVIATURAS}

CAIT: Centros de Atención Infantil Temprana • CDIAT: Centros de Desarrollo Infantil y Atención Temprana • GAT: Grupo de Atención Temprana.

5. Wechsler D. WPPSI-III. Escala de Inteligencia de Wechsler para preescolar y primaria-III. Madrid: TEA; 2009.

6. McCarthy D. MSCA. Escalas McCarthy de aptitudes y psicomotricidad para niños. Madrid: TEA; 2006.

7. Frostig M. Test de desarrollo de la percepción visual Frostig. Madrid: TEA; 2008.

8. Agencia de Calidad Sanitaria de Andalucía. Manual de estándares de los Centros de Atención Infantil Temprana; 2012. 